\title{
Keck Interferometer status and plans
}

\section{Mark Colavita, Peter L. Wizinowich, Rachel L. Akeson}

M. Mark Colavita, Peter L. Wizinowich, Rachel L. Akeson, "Keck Interferometer status and plans," Proc. SPIE 5491, New Frontiers in Stellar Interferometry, (20 October 2004); doi: 10.1117/12.552149

Event: SPIE Astronomical Telescopes + Instrumentation, 2004, Glasgow, United Kingdom 


\title{
Keck Interferometer status and plans
}

\author{
M. Mark Colavita ${ }^{\mathrm{a}}$, Peter L. Wizinowich ${ }^{\mathrm{b}}$, Rachel L. Akeson ${ }^{\mathrm{c}}$ \\ ${ }^{a}$ Jet Propulsion Laboratory, California Institute of Technology, \\ 4800 Oak Grove Dr., Pasadena, CA 91109 USA \\ ${ }^{\mathrm{b}}$ W. M. Keck Observatory, 65-1120 Mamalahoa Hwy, Kamuela, HI 96743 USA \\ ${ }^{c}$ Michelson Science Center, California Institute of Technology, 100-22, Pasadena, CA 91125 USA
}

\begin{abstract}
Keck Interferometer is a NASA-funded project to combine the two $10 \mathrm{~m}$ Keck telescopes for high sensitivity nearinfrared fringe visibility measurements, nulling interferometry at $10 \mu \mathrm{m}$ to measure the quantity of exozodiacal emission around nearby stars, and differential-phase measurements to detect "hot-Jupiters" by their direct emission. It is being developed by the Jet Propulsion Laboratory, the W. M. Keck Observatory, and the Michelson Science Center. Recent activity has included formal visibility mode commissioning, as well as science observations, and we briefly review some of the significant technical aspects and updates to the system. We have also completed laboratory development of the nuller. The nuller uses two modified Mach-Zehnder input nullers, a Michelson cross combiner, and a $10 \mu \mathrm{m}$ array camera to produce background-limited null measurements. To provide required temporal stability for the nuller, the system incorporates end-to-end laser metrology with phase referencing from two $2.2 \mu \mathrm{m}$ fringe trackers. The nuller recently completed its pre-ship review and is being installed on the summit. After nuller integration and test, the differential phase mode will be deployed, which will use a $2-5 \mu \mathrm{m}$ fringe detector in combination with a precision path length modulator and a vacuum delay line for dispersion control.
\end{abstract}

Keywords: interferometers, adaptive optics, nulling, differential phase

\section{INTRODUCTION}

The Keck Interferometer (KI) combines the two $10 \mathrm{~m}$ Keck telescopes in the following modes:

- High sensitivity fringe visibility $\left(V^{2}\right)$ measurements. This mode combines the adaptive-optics (AO) corrected beams from the two Kecks for measurement of fringe amplitude in the near-infrared.

- Infrared nulling at $10 \mu \mathrm{m}$. Using a nulling beam combiner to suppress on-axis light, this mode will be used to measure the quantity of zodiacal dust around nearby stars for studies of planetary formation and for source selection for the Terrestrial Planet Finder mission.

- Differential-phase interferometry. Using a precision fringe detector and simultaneous multi-color observations of fringe position in the near infrared, this mode will be used to detect the fringe shift caused by hot companions to nearby stars.

The fringe visibility mode has recently completed its Operations Readiness Review, while the nuller has just completed its pre-ship review and summit delivery. We will discuss these in more detail, below, along with updates on differential phase development and the status of the various subsystems. More detail on the system architecture and previous activity is found in [3] and references therein.

\section{2. $\mathbf{V}^{2}$ MODE}

\subsection{Science \& operations}

Shared-risk science observations in visibility mode with the Keck Interferometer began in June 2002. Shared-risk observers included 4 external teams, selected through a NASA Research Announcement; members of the internal 
development team; and TAC-selected observers within the CARA community. Astronomical sources observed have included young stellar objects: T Tauri stars, Herbig Ae/Be stars, and binaries; Wolf-Rayet stars; main sequence stars and binaries; and active galactic nuclei. See [1] for more detail on Keck Interferometer science.

To date, the shared-risk observations have resulted in two refereed science papers: on observations of the young stellar object DG Tau [2], and the galaxy NGC4151 [11] (see also [1], [10] at this conference); the NGC4151 observation was the first extra-galactic measurement with an optical/IR interferometer.

With the completion of an Operations Readiness Review in April 2004, the interferometer will be made available formally through the normal CARA-community TAC process.

The $\mathrm{V}^{2}$ Operations Readiness Review (ORR) marked the formal transition to operations for this mode. Similar reviews will be held for the nulling and differential phase modes after their development and validation is complete. However, completion of the $\mathrm{V}^{2}$ mode is an important milestone, as it validates a level of instrument performance and operational infrastructure which will be built upon by future modes. Details of the ORR are described elsewhere at this meeting [14]: the highest-level requirements for the review addressed $\mathrm{V}^{2}$ accuracy at a certain magnitude $(5 \%$ at $\mathrm{K}=9)$; interferometer and facility uptime (90\% and $80 \%$ ); and the operational infrastructure to conduct $\mathrm{V}^{2}$ observations.

\subsection{Instrument performance}

\section{Accuracy}

A requirement for the ORR was to demonstrate $\mathrm{V}^{2}$ accuracy, not just precision. We chose to approach this by observing binary stars for which good orbits had been determined from Palomar Testbed Interferometer data and radial-velocity measurements. The stars were then attenuated appropriately for the three KI operations bands: K (for fringe tracking), J (for angle tracking), and R (for adaptive optics). Neutral-density filters (rather than irises) were used to properly maintain the spatial properties of the wavefront. An example is shown in Fig.1, and more detail is provided in [14].
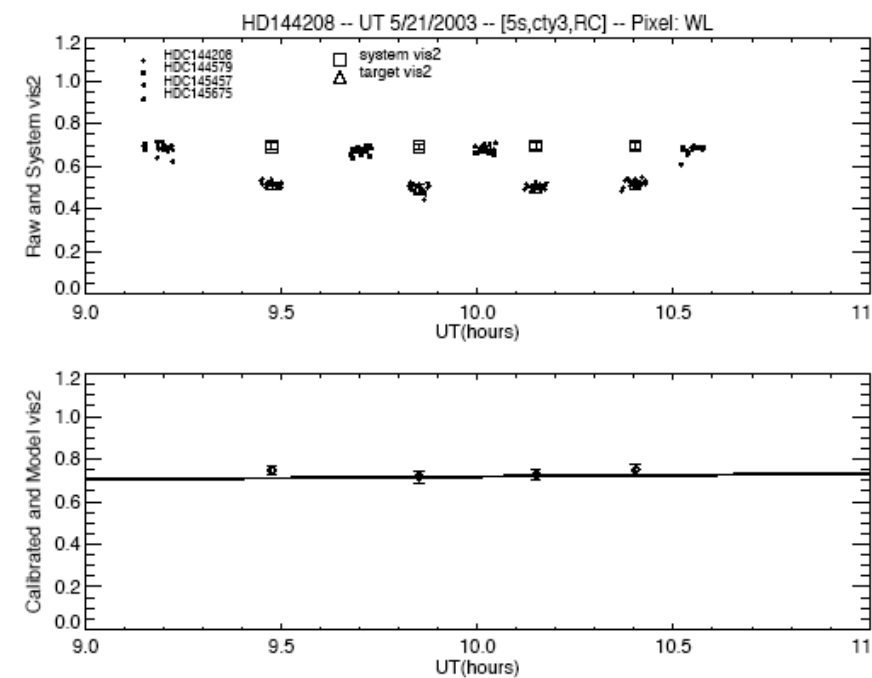

\begin{tabular}{|c|c|c|c|c|}
\hline \multicolumn{4}{|c|}{ Reduced mags: K:8.0, J:4.5, R:5.7 } & \\
\hline channel & $\begin{array}{l}\text { avg formal } \\
\text { error per scan }\end{array}$ & $\begin{array}{c}\text { reduced } \\
\chi^{2}\end{array}$ & <Obs-Model> & $\begin{array}{c}<0-M> \\
\text { uncertainty }\end{array}$ \\
\hline WL & 0.025 & 0.9 & 0.027 & 0.012 \\
\hline
\end{tabular}

Fig.1. $V^{2}$ validation data - intermediate magnitude case

The upper left panel shows target and calibrator observations: the square symbols are the mean calibrations for the science observations. The lower left panel shows the calibrated data, at one point per scan, along with the prediction. For this set, the mean deviation of the data from the model was 0.027 , with a formal uncertainty of 0.012 .

\section{Uptime and scans per hour}


System uptime was tracked carefully, both to verify compliance with ORR requirements, as well as to aid in addressing problems and improving overall performance. The system uptime met or exceeded requirements over the interval preceding the ORR (Fig. 2). Scans per hour was also tracked carefully; results are also presented in Fig. 2, which shows an average rate over all usable time of greater than 4 scans per hour (each scan is $125 \mathrm{sec}$ of fringe data and associated detector calibrations), with peak rates of better than 6 scans per hour.
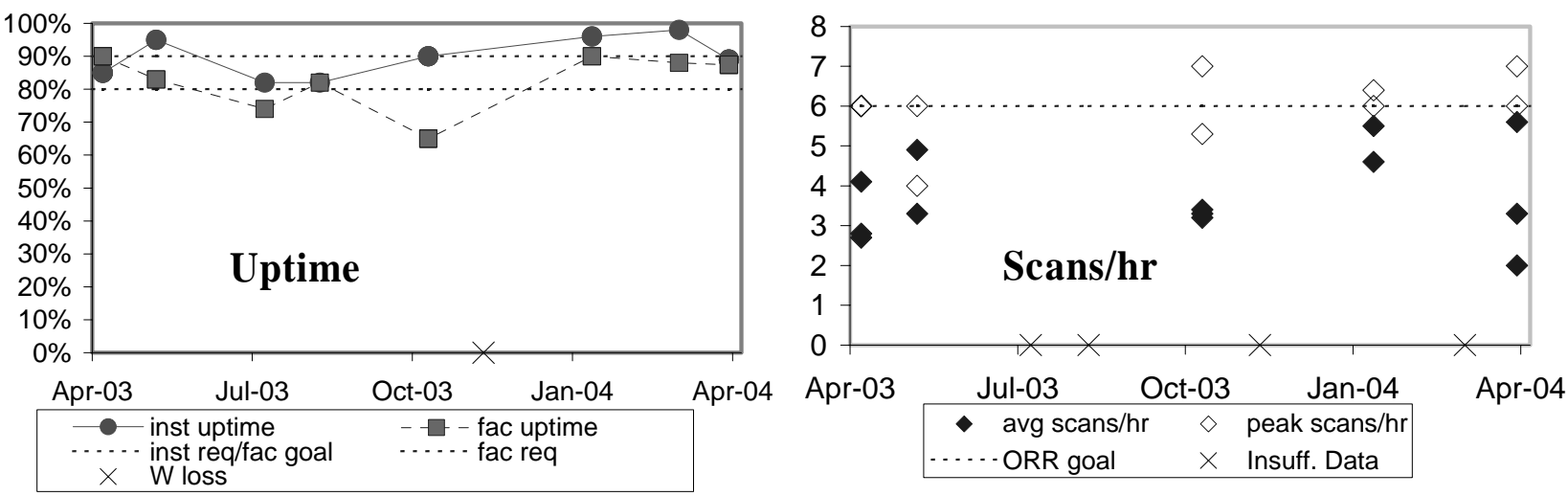

Fig. 2. Uptime and scans per hour performance

\section{Sequencing}

KI uses post-combination single-mode fibers to feed the fringe tracker. While simultaneous flux measurements of the individual arms are not available, knowledge of the average flux ratio over the measurement interval can be used to correct the average visibility for reductions attributable to unbalanced intensities [9]. However, analysis of KI data indicates that the coupled intensity includes a $1 / \mathrm{f}$ component down to frequencies less than $0.1 \mathrm{~Hz}$. This low-frequency energy is such that the intensity ratio determined from an end-of-scan measurement of the fluxes of the individual apertures may differ from the actual ratio during the integration time. Thus, we interleave a series of short singleaperture flux measurements throughout the integration time, and compute the flux ratio for the scan from the ratio of the average single-aperture fluxes. In the presence of low frequency fluctuations, modeling indicates that such an approach yields a greatly-improved estimate, both with respect to systematic bias $(<<1 \%)$ as well as statistical noise (approx. $2 \times$ quieter). We typically interleave $5 \mathrm{sec}$ measurements on each aperture every $25 \mathrm{sec}$.

Another observation from operational experience is that the fiber coupling, despite stable alignment and inclusion of an atmospheric dispersion compensator in the angle tracker, can still be improved by on-star optimization of the flux. Thus, each scan typically includes a fiber-coupling optimization step. For this, we use a raster mirror in the fringe tracker feed optics to scan the star's image across the fiber tip, and then offset the angle tracker to provide a new pointing offset.

\section{Phase and amplitude stability}

With respect to phase stability, we generally achieve our requirement of $<70 \mathrm{~nm}$ rms vibration in any 4-ms window, corresponding to a $4 \% \mathrm{~V}^{2}$ reduction (largely calibratable). We compute this metric, which we have been tracking over the past two years, using a filter function applied to the power spectrum of the fringe-tracker residual, after subtracting the seeing component. We achieve this level of performance through the use of end-to-end laser metrology feedback to the fast delay line, which controls the end-to-end path from the fringe tracker to the dual-star module, as well as with feedforward of telescope piston. However, for typical frame times the metrology provides the largest improvement. 
There have also been improvements in a number of other areas, including passive (e.g. AO bench stiffening) and active measures (e.g. delay line servo improvements), discussed under the specific subsystems, below.

With respect to amplitude stability, we have also made improvements in several areas, both passive (seeing improvements) and active (HHC controller in the angle tracker), as discussed below. These are in addition to the changes to the observational scenario described above.

The current instrument phase and amplitude stability performance meets our requirements for $\mathrm{V}^{2}$ mode and our current error-budget allocations for nulling and differential phase modes, which observe relatively bright sources. However, the current performance is not at fundamental limits, and we have identified areas where further investigation is warranted, or specific improvements could be implemented. The stability performance will be reexamined after we have on-sky data to validate the nuller and differential-phase error budgets.

\section{SUBSYSTEM UPDATES}

Continued development of the interferometer has proceeded since the last update [3]; some highlights are given below.

\section{Adaptive optics}

A number of general operational and performance improvements have been made to the Keck I and II AO systems, as discussed in [15]. These include: conversion of the AO enclosures into clean rooms, placing the AO electronics on UPS, upgrades of the AO host computers, a port of the wavefront controller command processor from Solaris/SPARC to EPICS/PowerPC, implementation of a new operator's user interface, recoating and realignment of the Keck I and II image rotators, and implementation of higher resolution and larger range field steering mirrors. AO characterization and optimization efforts [4] have included studies of wavefront sensor spot size, optimal focusing of the wavefront sensor camera, system interaction matrices, tip/tilt residuals, control loop parameters, centroid origins and how they are affected by SNR, centroid gains and how they are affected by spot size, subaperture selection, reconstruction matrix, CCD noise, flat fielding, etc. Performance improvements have been demonstrated with the implementation of optimized calibration routines, a better system matrix and tip/tilt and deformable mirror gains, smarter reconstruction matrices (Bayesian), a fix to the interaction matrix generation code, CCD flat-fielding, centroid origin scaling for extended objects or bad seeing, etc. In particular, the new reconstruction matrices have been responsible for a $10 \%$ Strehl increase. More detail is available in [15].

Interferometer-specific modifications to the $\mathrm{AO}$ systems on $\mathrm{K} 1$ and $\mathrm{K} 2$ include the implementation of chopping with the $\mathrm{AO}$ tip/tilt mirror to support the nuller mode. Mechanical modifications were also made to the mounting of the dichroic that extracts light from the collimated space after the deformable mirror and directs it to the interferometer beam train. This mount is not monitored by the internal metrology, and changes were made to its motion-control stage to provide a stiffer insertion position (the optic is removed from the beam for other uses of the AO system).

\section{Dual-star module}

The dual-star modules (DSMs) have seen only minor changes from last time. Most of the changes in support of $\mathrm{V}^{2}$ mode have addressed improved remote controls for alignment. In addition, the DSMs are now in the AO clean rooms, discussed above, which helps maintain optical quality.

Larger DSM changes will occur for the nulling mode, as the DSM will provide the pupil splitting as required for the nuller implementation. In this mode, the left and right halves of the Keck pupil will be directed down the primary and secondary coude trains toward the basement. These changes will be implemented with motion-control stages to allow reverting the configuration to the $\mathrm{V}^{2}$ mode. Additional DSM changes will include the incorporation of virtual cold aperture stops for nuller mode for reduced background, as well as a reconfiguration of the metrology pickoff: the approach used for $\mathrm{V}^{2}$, which extracts the metrology through a hole in a mirror in the shadow the telescope secondary, will be replaced by one which uses a visible-transmissive dichroic to avoid any beam obscuration with the split pupils. 


\section{Coude train}

The primary beam from each telescope, as is used for $\mathrm{V}^{2}$, follows a nominally passive coude path, with the final downward fold along the azimuth axis. However, for the secondary beam, the coude path must be active to maintain a fixed pupil and beam direction at the base of the telescope (alternatively, the primary and secondary beams could be arranged adjacent to each other and steered passively along the azimuth axis; however large coude optics would be required, and also a large rotator at the base of the telescope to correct for the pupil rotation). The secondary coude system is implemented using off-axis tracking mirrors at the M6S (top of azimuth axis) and M7S (base of telescope) locations: M6S tracks azimuth, steering the pupil onto M7S; M7S then steers to maintain a fixed output direction, parallel to the primary beam. Control of this secondary system is implemented using EPICS, and has been tested in support of nuller operations. Thus, at the output of the coude train, there are two parallel beams, which then share common transport optics through the long delay lines before being split to feed the fast delay lines.

\section{Long delay lines \& auto-alignment}

Since last time, the Long Delay Lines (LDLs) have had their motion control systems installed, allowing remote reconfiguration, and in particular, multiple reconfigurations during a night, which greatly increases the available sky coverage. The motion control system includes tractor motors, cable tensioners, alignment actuators, and the associated drivers and control system. As the long delay lines are implemented with flat mirrors to minimize reflections, the angle of the mirrors must be trimmed after completing a move. To automate this process, an auto-alignment system is used. The sensor for the system is a back-end visible-wavelength camera that views pop-up LED targets along the beam train. For simplicity, the back-end camera is fixed focus (rather than a formal alignment telescope), and is supported by centroiding software that can accommodate a defocused image.

A realignment of the beam train restores both the transverse pupil location and the beam direction. The current alignment procedure after an LDL move restores target centroid values to their pre-move values by moving the preceding optic in the beam train (after a coarse positioning based on a lookup table). Fine-tuning of the angular alignment - the more critical of the two alignments, given the 0.6" FOV (on the sky) of the angle tracker - uses the image of the AO stimulus on the angle tracker itself. More detail on the auto-alignment system and its testing is given in [5].

\section{Fast delay lines}

The Fast Delay Lines (FDLs) have not changed so much as multiplied. While $\mathrm{V}^{2}$ mode requires just two FDLs, full-up nulling mode requires eight. Four FDLs are currently in place on the summit, while the remaining four are at JPL, and will ship out in support of the nuller. The only notable changes have occurred in the control algorithm, which has had minor upgrades for improved tracking and error rejection. The FDLs have been formally handed over to CARA for all maintenance and future development.

\section{Fringe tracker}

The internal optics for the fringe tracker [13] have been updated to add an additional set of direct-view prisms, to enable all four dewar inputs, and to provide improved encircled energy. In addition, the internal alignment PZTs have been enabled: as the dewar input is via single-mode fiber feedthroughs through the dewar wall, the PZTs allow fine tuning of the images onto the array such that they fall on a single pixel (white-light side) or a single line (spectrometer side).

Alignment along the dispersion direction is achieved using the second spectrometer PZT and a narrow-band filter in the stimulus white-light path which provides a reference wavelength.

The fringe tracker routinely operates with a 5-ms frame time, and with addition of the second prism set, the fringe tracker provide H-band capability in addition to its K-band capability. The fringe tracker implements a phase delay / group delay controller for high-band width fringe tracking / low-bandwidth fringe centering. Some improvements were made to the synchronization of the fringe-tracker readout with respect to the delay-line OPD modulation, which resulted in a reduction in visibility systematic errors. In addition, a low-frequency target dither was added to the group delay 
loop, such that the fringe track point is (approximately) uniformly distributed over $2 \pi$. For the case of simple modulation-amplitude mismatches, this changes the dependence of the systematic error in visibility on relative mismatch from a linear phase-dependent bias to an approximately phase-independent quadratic bias, further improving the data quality. Finally, a minor change was made to the clock pattern, which results in greatly reduced settling time to a stable calibration after initiation of array clocking.

As discussed above, the fringe-tracker fore optics now includes a raster mirror which is used for optimizing the coupled flux. Also, the second fringe-tracker foreoptics table was installed, as required for use with the nuller. This foreoptics table feeds the remaining two (of four) fiber inputs into the camera dewar. New clock patterns to read out the four regions of interest on the array were written and tested.

\section{Angle tracker}

The angle tracker routinely operates at $50 \mathrm{~Hz}$ and $\mathrm{J}$ band. Other rates $(100 \mathrm{~Hz}, 20 \mathrm{~Hz}$ (and slower)) are provided, and $\mathrm{H}$ band is also supported (although with reduced throughput, as most of the $\mathrm{H}$ band is reserved for the fringe tracker with the current dichroic set). To allow J-band operation at high zenith angles (the J-K angular dispersion is approximately two K-band diffraction spots at a zenith angle of 45 degrees), a differential atmospheric dispersion compensator has been incorporated. It is implemented with a pair of Risley prisms in the nearly-collimated common input to the angletracker camera, so that it corrects both input beams.

We have also modified the angle-tracker control algorithm to suppress a narrow-band disturbance originating on one of the telescope secondaries that was causing fluctuations in the fringe-tracker intensity. While observable on our angle tracker at a $100 \mathrm{~Hz}$ sampling rate, the high frequency precluded standard servo suppression. However, because of the narrow-band nature of the disturbance, synchronous detection of the disturbance at the output of the angle tracker was implemented to tune an oscillator that is summed with ordinary tip-tilt mirror commands to cancel the vibration. The approach is very similar to the HHC ("higher harmonic control") approach. An interesting feature is that it can be used for disturbances above the Nyquist frequency (with appropriate gain, and sign of gain, changes), and we implement it for $29 \mathrm{~Hz}$ suppression even at our 50-Hz sample rate.

\section{Real-time control system and MSC tools}

The real-time control system, both RTC-Toolkit- and EPICS-based, orchestrates most of the functional updates described above in the context of specific subsystems. In addition, the interferometer sequencer has been significantly updated since last time, with a new infrastructure and additional functionality to support the current observational scenario; more detail on the interferometer sequencer is available in [7]. An EPICS-based telescope sequencer been also been added since last time, which in coordination with the interferometer sequencer, controls telescope slewing and AO-system acquisition. New GUIs are also available for the RTC-Toolkit systems that can be easily built or updated by the operator.

The Michelson Science Center (MSC) tools [6] have also been updated: notable is a near real-time version the $\mathrm{V}^{2}$ processing software. This software runs during observations, providing rapid feedback on instrument performance (total intensity, intensity balance, tracking jitter, etc.), as well quick-looks at the measured visibility.

\section{Facility improvements}

For the facility, the dewar auto-fill system has been completed for the fringe and angle tracker cameras, and extended to support the LN2 fills required for the nulling and differential phase modes. Various air conditioners have been vibration isolated, which allows them to be run during the night (rather than being replaced by fans) without observable vibration in the interferometer. Also, a number of seeing improvements were made in the basement, including an air-flow survey leading to the reduction of some air leaks; insulation of the coude pipes from the basement to the telescope crypt, and insulation of the crypts themselves. 


\section{UPCOMING MODES}

\subsection{Nuller}

The nuller completed its pre-ship review on June 9, 2004, in preparation for summit delivery and summit integration and test; more details are available in [8]. The nuller uses a two-step approach to conduct its measurements: as shown in Fig. 3a, two modified Mach-Zehnder nullers null the light from the left and right halves of the two telescopes, resp., to suppress the central star. These nulled outputs are then directed into a cross combiner, which is a conventional Michelson combiner, and the combined light detected by a 10- $\mu \mathrm{m}$ array camera using a 4-bin algorithm. Fig. $3 \mathrm{~b}$ shows a bit more detail of the system architecture as will be implemented on the summit. The key additional feature indicated in the figure is the use of the $2.2 \mu \mathrm{m}$ fringe trackers for high speed path-length stabilization.

For final laboratory integration and test, a fairly complete test setup was assembled in the lab at JPL, including the nuller breadboard (two modified Mach-Zehnder nullers + cross combiner); a stimulus breadboard (containing whitelight, and infrared- and visible-laser sources); the infrared camera; a switchyard for separating $\mathrm{K}$ and $\mathrm{N}$ band light, including retro-flats; two $2.2 \mu \mathrm{m}$ fringe trackers; 8 delay lines ( 4 active and 4 (temporary) passive); internal metrology; and the real-time control system for automated nuller and cross combiner fringe acquisition and tracking. The laboratory configuration is shown in Fig. 4.

In stand-alone configuration (retro-flats on the nuller breadboard itself; no metrology), the nuller has achieved shortterm white-light nulls of better than 10,000:1. In the end-to-end configuration described above, the nuller achieves stabilized nulls over several minute timescales of better than 1000:1. At this level of performance, and with completion of other requirement testing, we are ready of the next stage of testing at the summit. 

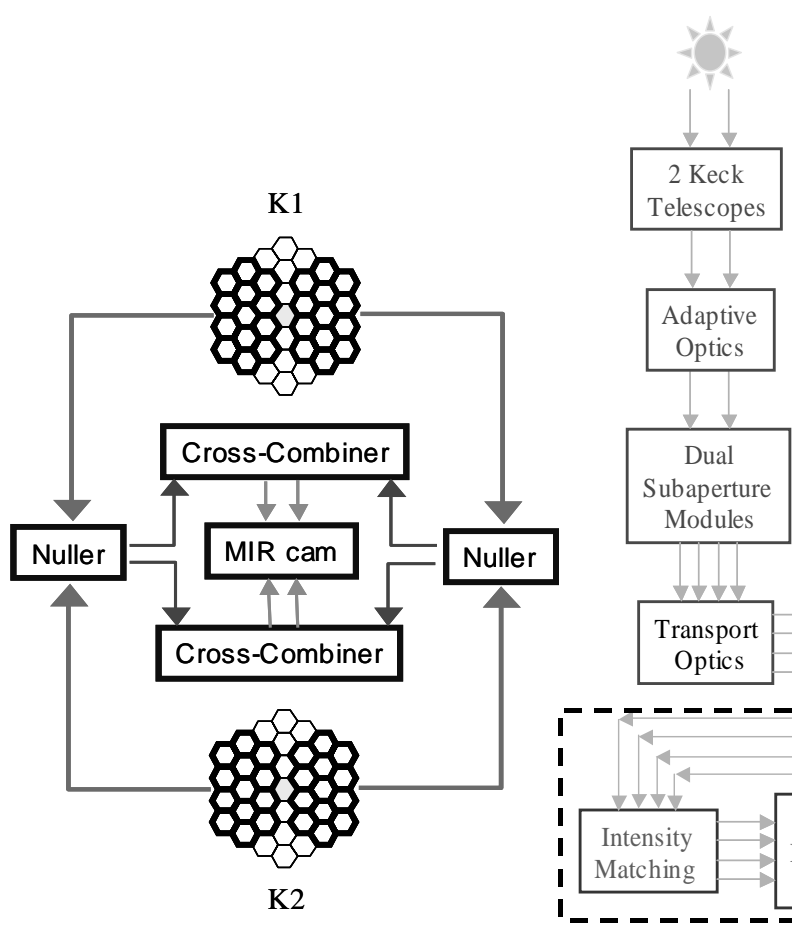

Fig. 3. (a) Nulling approach; (b) Nuller beam train schematic

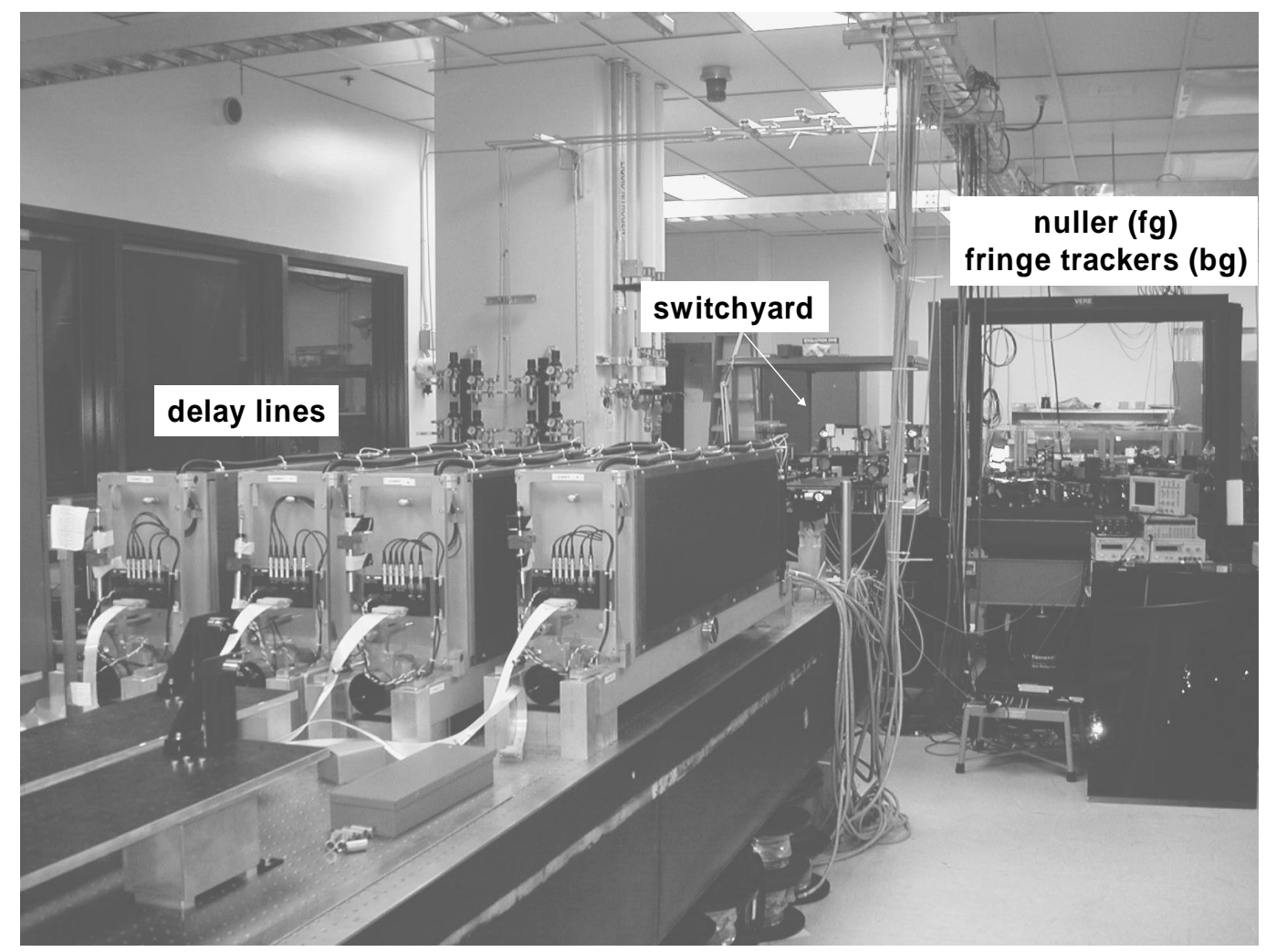

Fig. 4. Nuller laboratory integration and test setup 


\subsection{Differential Phase}

Development of differential phase (DP) subsystems has proceeded in parallel with nuller development. In particular, the KL camera, including the dewar, chalcogenide fibers, internal optics, and mid-wave PICNIC array detector for the 2-5 $\mu \mathrm{m}$ band has been assembled and tested. The KL beam combiner has been assembled and aligned. The voice-coil modulator and precision metrology has been tested. The first delay-cart for the differential-phase delay line has been assembled. And finally, the vacuum tubes for the DP delay lines have been installed at the summit. With the shipment of the nuller hardware to the summit, integration of the differential phase subsystems will now begin in the lab at JPL. Summit integration of the DP will follow summit integration of the nuller. More detail on differential phase is available in [12].

\section{ACKNOWLEDGEMENTS}

The Keck Interferometer is funded by the National Aeronautics and Space Administration (NASA). Part of this work was performed at the Jet Propulsion Laboratory, California Institute of Technology, and at the Michelson Science Center, California Institute of Technology, under contract with NASA.

\section{REFERENCES}

[1] R. L. Akeson 2004, “Keck Interferometer Science: Present and Future,” Proc. SPIE, 5491 (this conference)

[2] M. Colavita, R. Akeson, P. Wizinowich, et al. 2003, "Observations of DG Tauri with the Keck Interferometer," ApJ, 592, L83

[3] M. M. Colavita \& P. L. Wizinowich 2003, "Keck Interferometer Update,” Proc. SPIE, 4838, 79

[4] M. A. van Dam, D. Le Mignant, \& B. A. Macintosh 2004, "Performance of the Keck Observatory Adaptive Optics System," Appl. Opt., in press

[5] M. Hrynevych, K. Tsubota, R. Smythe, et al. 2004, "Keck Interferometer Autoaligner: Algorithms and Techniques," Proc. SPIE, 5491 (this conference)

[6] http://msc.caltech.edu/KISupport/index.html

[7] L. J. Reder, A. Booth, \& J. Hsieh 2004, "Development of a State Machine Sequencer for the Keck Interferometer," Proc. SPIE, 5496 (this meeting)

[8] E. Serabyn 2004, “The Keck Interferometer Nuller,” Proc. SPIE, 5491 (this conference)

[9] S. B. Shaklan, M. M. Colavita, \& M. Shao 1992, ESO Conf. and Workshop Proc., 39, 1271

[10] M. R. Swain, 2004, “Observing NGC 4151 with the Keck Interferometer,” Proc. SPIE, 5491 (this conference)

[11] M. Swain, G. Vasisht, R. Akeson, et al. 2003, "Interferometer Observations of Subparsec-Scale Infrared Emission in the Nucleus of NGC 4151," ApJ, 596, L163

[12] G. Vasisht \& M. M. Colavita 2004, "Differential Phase Interferometry with the Keck Telescopes," Proc. SPIE, 5491 (this conference)

[13] G. Vasisht, A. J. Booth, M. M. Colavita, et al. 2003, "Performance and Verification of the Keck Interferometer Fringe Detection and Tracking System,” Proc. SPIE, 4838, 824 
[14] P. Wizinowich, R. Akeson, E. Appleby, et al. 2004, "Visibility Science Operations with the Keck Interferometer," Proc. SPIE, 5491 (this conference)

[15] P. Wizinowich et al. 2004, "Adaptive Optics Developments at Keck Observatory," Proc. SPIE, 5490 (this meeting) 